\title{
An End-to-End Delay-based Scheduling Algorithm in IEEE 802.15.4e Networks
}

\author{
Yang G. Kim ${ }^{1}$, ByoungSeob Park ${ }^{2}$ and Hyo Hyun Choi ${ }^{3 *}$ \\ ${ }^{1}$ Department of Computer Engineering Technology, New York City College of \\ Technology of the City University of New York, 300 Jay Street, Brooklyn, NY, USA \\ ${ }^{2}$ Department of Computer Systems \& Engineering, Inha Technical College, 100 \\ Inha-Ro, Namu-Gu, Incheon 402-752, Republic of Korea \\ ${ }^{3}$ Department of Computer Science, Inha Technical College, 100 Inha-Ro, Namu- \\ $\mathrm{Gu}$, Incheon 402-752, Republic of Korea \\ Ifixcom@hotmail.com, ${ }^{2}$ bspark@inhatc.ac.kr, ${ }^{3}$ hchoi@inhatc.ac.kr
}

\begin{abstract}
This paper proposes a novel scheduling algorithm using multi-channel and multitimeslot with the objective of reducing the end-to-end $(e 2 e)$ delay in a tree-based wireless sensor network. The algorithm exploits a staggered timeslot allocation in terms of e2e paths, rather than the one with individual branches. It also increases simultaneous data transmissions. The proposed algorithm is shown to be superior to an existing algorithm with non-e2e delay based, in terms of e2e delay.
\end{abstract}

Keywords: Multi-channel, multi-timeslot, multi-hop, scheduling algorithms, end-to-end delay, wireless sensor networks

\section{Introduction}

Wireless sensor networks (WSNs) are flourishing due to their various applicationcapable characteristics. Wireless sensors, which were initially developed to simply detect and react, have become communication-capable sensors, collecting and forwarding sensed data towards a data collector. Data collection in large-scale applications, such as bridge monitoring, environment monitoring, and intruder detection would require multi-hop communication because the sensors are deployed in large areas. Time-critical applications, such as patient monitoring, disaster warning, and intruder detection, and many industrial applications require expedited delivery of data, because belated data from just one source of data may render the entire system useless.

The traffic in the industrial applications is usually periodic, making Time Division Multiple Access (TDMA) approach feasible. Each TDMA timeslot is extended to accommodate multiple channels as in the IEEE Standard 802.15.4e [1] to guarantee e2e delay. Then, each node has the flexibility of utilizing a combination of channel and timeslot in order to achieve expedited data packet transmission towards the data collector, resulting in minimum e2e delay.

The IEEE 802.15.4e standard is a global standard for industrial wireless sensor networks applications, including smart grid, telemedicine, process automation, and factory automation. It supports these industrial applications with deterministic latency, high reliability, and flexibility in multi-hop QoS WSNs [2]. Application scenario in IEEE 802.15.4e is an example of where the delay for individual reading is more important than the delay for collecting all the data from all the nodes in the network (i.e., average delay).

There are inherently two factors that hinder efficient data collection in WSNs: halfduplex nature of transceiver and interference [3]. In WSNs, each sensor node is typically

* Corresponding Author 
equipped with a single half-duplex radio transceiver, i.e., each node cannot transmit and receive simultaneously, nor can it function on different channel at the same time. In a tree topology, the data traffic typically flows from a child (transmitter) to a parent (receiver) towards the coordinator. Since interference occurs at the receiver end, i.e., the parent, in order to cope with interference, the channel of each parent node that experiences interference is changed starting with the parent with the most number of interfering nodes.

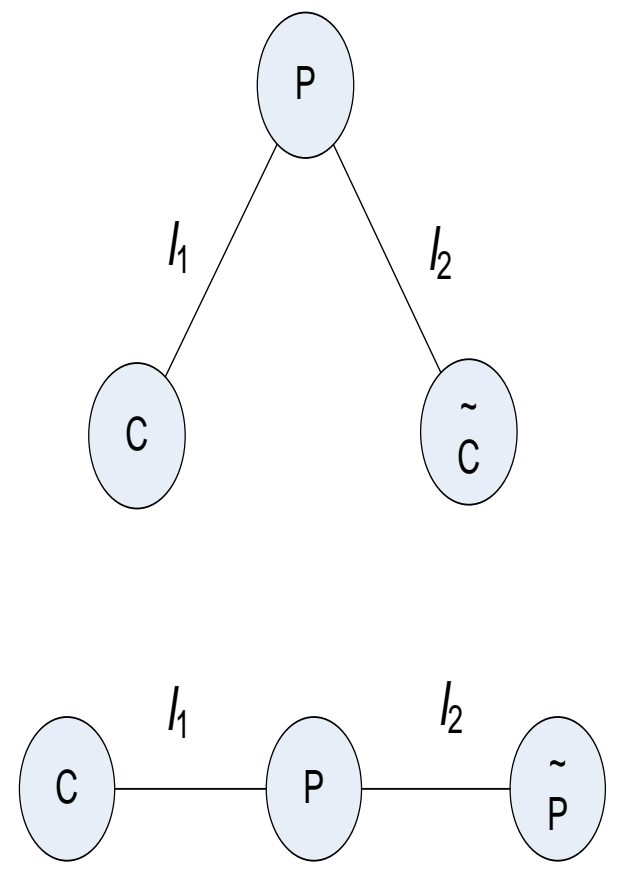

a) adjacency link

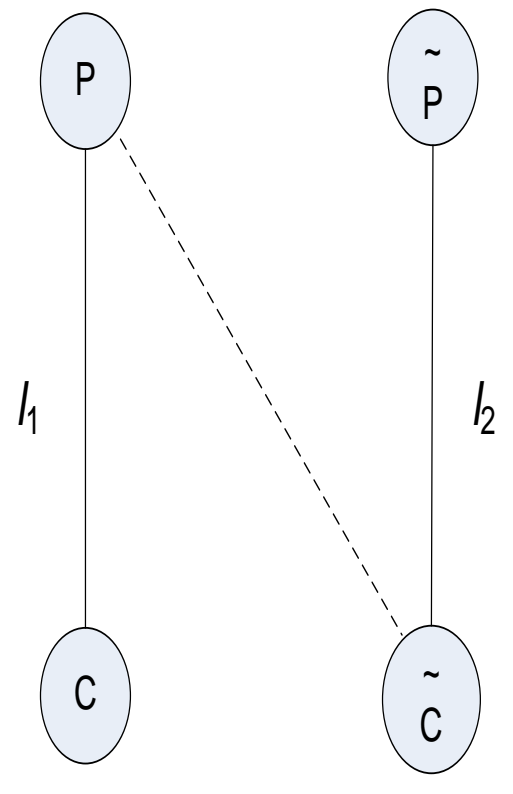

b) interference link

\section{Figure 1. The Definition of Constraints (Solid Lines are Communication Links while a Dotted Line is Interference Link)}

Interfering parents are defined as a pair of parents $p$ and $\tilde{p}$ where the transmission from the child $\tilde{\mathrm{c}}$ to its parent $\tilde{\mathrm{p}}$ interferes with the transmission from the child $\mathrm{c}$ to its own parent $p$, and vice versa. Formulating collision-free TDMA schedules has been proved to be an NP-complete problem [3], [4]. The adoption of multiple channels into each TDMA timeslot makes the scheduling even more challenging. In our previous work [5], we apply the metaheuristic optimization to schedule the resources with a given end-to-end delay bound in mind. Then, any solution that results in an e2e delay lower than the required delay bound is acceptable. However, although there would be a trade-off to relax the computation time at the expense of the solution quality, the optimization algorithm will still be a burden to resource-constrained WSNs. We propose a simple/greedy heuristic scheduling algorithm for channel and TDMA timeslot allocations to minimize the e2e delay.

\section{Network Model and Problem Formulation}

We model the multi-hop WSN as a graph $\mathrm{G}=(\mathrm{V}, \mathrm{E})$, where $\mathrm{V}$ is the set of nodes, and $E=\{(i, j) \mid i, j \in V\}$ is the set of edges representing the wireless links. $A$ designated node $s \in \mathrm{V}$ denotes the sink. The Euclidean distance between two nodes, $i$ and $j$, is denoted by $d_{i j}$. Each node in the tree topology generates a single packet, 
and each intermediate node would have two roles: a sender and a forwarder. The packets from all the nodes are destined to the coordinator, which is the typical scenario for data collection in WSNs. Our objective is to minimize e2e delay for each node generating and forwarding packets towards the coordinator while considering the following two constraints:

- Adjacency constraint: two edges $(\mathrm{i}, \mathrm{j}) \in \mathrm{E}$ and $(\mathrm{k}, \mathrm{l}) \in \mathrm{E}$ cannot be scheduled in the same timeslot if they are adjacent to each other, i.e., $\{\mathrm{i}$, $\mathrm{j}\} \cap\{\mathrm{k}, 1\} \neq \varnothing$ due to the half-duplex transceiver in Figure 1 (a).

- Interfering constraint: two edges $(\mathrm{i}, \mathrm{j}) \in \mathrm{E}$ and $(\mathrm{k}, \mathrm{l}) \in \mathrm{E}$ cannot be scheduled simultaneously if $(i, j)$ or $(k, 1)$ is an interference link in Figure 1 (b).

\section{E2E Delay-based Channel and Timeslot Scheduling}

A) Existing approach (non-e2e delay-based): Incel et al., [3] studies two separate scheduling problems: scheduling for multi-channel at a receiver side and multi-timeslot at a transmitter side. For non-e2e based approaches, the solution provided in [4] has been proven to be the optimal in determining the minimum number of timeslots required for data transmission, rather than the e2e delay. The receiver-based channel scheduling assigns the channels to the receivers (i.e., parents) in order to eliminate all the interfering links at a receiver side. All potential receivers calculate the amount of interferences in terms of the SINR. If the SINR of a transmission from child $\mathrm{c}$ to its parent $\mathrm{p}$ is less than the SINR of the interfering signal from interfering child $\tilde{\mathrm{c}}$ to parent $\mathrm{p}$, the parent $\tilde{\mathrm{p}}$ is said to be interfering with parent $\mathrm{p}$ as in Figure 1 (b). All interferences experienced by all interfered parents in the tree are eliminated by allocating appropriate channels for all the interfered parents. The channel assignment starts with the parent with the most number of interfering nodes. If the number of available channels is not enough to eliminate all interferences, the remaining interferences are eliminated by allocating timeslots at the transmitters (child nodes), thereby transmitting data packet on the designated timeslot using the same channel.

Once the channel assignment is completed at a receiver side to remove potential interferences, timeslot allocation is performed at the transmitter side to increase parallel transmission along multiple branches. If a branch with the highest number of remaining nodes/packets is to be scheduled [3], [4], the timeslot allocation for the node at the top of the branch should be with consecutive timeslot numbers. For example, in Figure 2, the node 2 requires three timeslots: a sender and two forwarders. Then, the node 2 would be assigned three consecutive timeslots, 1, 2, and 3, which would increase the e2e delay for the node 3 and 4, because their paths are neither weighed nor treated equally in terms of e2e delay. 


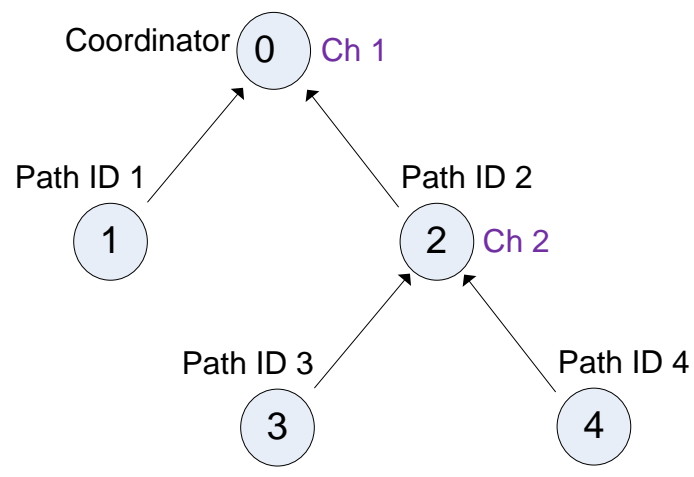

\begin{tabular}{|c|c|c|c|c|}
\hline Path & \multicolumn{3}{|c|}{ Node } & Hop \\
\hline 1 & 1 & & & 1 \\
\hline 2 & 2 & & & 1 \\
\hline 3 & 3 & 2 & & 2 \\
\hline 4 & 4 & 2 & & 2 \\
\hline
\end{tabular}

\section{non-e2e delay-based}

\begin{tabular}{|c|c|c|c|c|c|c|}
\hline \begin{tabular}{|c|c|c|}
\hline Patheslot \\
Path
\end{tabular} & 1 & 2 & 3 & 4 & 5 & $\begin{array}{c}\text { e2e } \\
\text { delay }\end{array}$ \\
\hline Path 1 & 1,1 & & & & & 1 \\
\hline Path 2 & & 2,2 & 3,2 & 4,2 & & 1 \\
\hline Path 3 & & & & & 3,3 & 4 \\
\hline Path 4 & 4,4 & & & & & 4 \\
\hline
\end{tabular}

\section{e2e delay-based}

\begin{tabular}{|c|c|c|c|c|c|c|}
\hline $\begin{array}{c}\text { Tatheslot } \\
\text { Path }\end{array}$ & 1 & 2 & 3 & 4 & 5 & $\begin{array}{c}\text { e2e } \\
\text { delay }\end{array}$ \\
\hline Path 1 & 1,1 & & & & & 1 \\
\hline Path 2 & & & & & 2,2 & 1 \\
\hline Path 3 & & & 3,3 & 3,2 & & 2 \\
\hline Path 4 & 4,4 & 4,2 & & & & 2 \\
\hline
\end{tabular}

\section{Figure 2. The Delay Comparison of the Non-e2e delay-based and e2e Delay-based Algorithms}

B) Proposed approach (e2e delay-based): As with the receiver-based channel scheduling [3], the algorithm first determines interfering parents by using with the SINR values. Assume each node has eight different transmission power levels $(\mathrm{P})$, and the value of SINR refers to the amount of interference to neighboring parents [3]. Each node in a tree has its own node vector (path ID, node ID) for the transmission of a packet to the coordinator. The node ID of each node is the same as the path ID of the path starting from that node and ending at the coordinator (e.g., the node 4 has a path, which starts from node 4 via node 2 to the coordinator). Once the channel assignment has been completed at a receiver side, timeslot allocation is 
performed at the transmitter side to increase parallel transmission along multiple paths. In a given timeslot, there would be more than one eligible path in a tree topology because the coordinator can receive data packet from one path among many. Then, a path with the longest path-length should be scheduled first because each node has a different remaining path-length to the coordinator. The e2e delaybased algorithm schedules timeslots starting from leaf node for the first available timeslot to the intermediate node for next available timeslot in a staggered way towards the coordinator. The major distinction between the non-e2e delay-based [3], [4] and the e2e delay-based algorithms is that the former schedules timeslots along the branches while the latter does so by the paths because each node has its own path towards the coordinator.

Illustration of proposed algorithm: In Figure 2, there are five nodes including the coordinator and three available channels. The parent nodes are the coordinator and 2 while the child nodes are node 1, 3 and 4 . After calculating interfering parents for each parent node, the coordinator has the most interfered parent and then it is assigned the channel number 1 , and the next interfered one is the node 2 , which is assigned the channel number 2. Note that parallel transmission happens at the first timeslot for node 1 and 4 with their different receiver channels, channel number 1 (coordinator) and channel number 2 (node 2). In view of the e2e delay, non-e2e delay-based algorithm [3] incurs four units of delay for path 3 and 4 due to the path sequence required, such as 3,3,3,2 and 4,4, 4,2, respectively, in a repeated frame, while e2e delay-based algorithm incurs two units of delay for path 3 and 4 . Note that both algorithms achieve the maximum timeslot number 5 due to the parallel transmission.

Notations:

$$
\left\{\begin{array}{c}
R_{n, \tilde{n}}: \text { a set of all potential paths }\left(r_{n, \tilde{n}}\right) \\
d_{r_{n, \tilde{n}}}: \text { the delay of a path between source } n \text { and destination } \tilde{n} \\
l, \tilde{l}: \text { the link between node } l \text { and } \tilde{l} \text { in a path } r_{n, \tilde{n}} \\
d_{l, \tilde{l}}: \text { the link delay for } l, \tilde{l}
\end{array}\right.
$$

The goal of the scheduling is to minimize the e2e delay, which follows:

$$
\mathrm{D}_{\mathrm{e} 2 \mathrm{edelay}}=d_{r_{n, \tilde{n}}}=\min _{r_{n, \tilde{n}} \in R_{n, \tilde{n}}}\left(\sum_{l, \tilde{l}} d_{l, \tilde{l}}\right)
$$

\section{Performance Evaluation}

We compare the performances of the non-e2e delay-based and e2e delay-based algorithms for each of 4 network sizes, 25, 50, 75, and 100 nodes, but we show 2 network sizes, 25 and 100 nodes due to space budget. The simulation is carried out on a $500 \mathrm{~m} \times 500 \mathrm{~m}$ field for 25 and 50 nodes, and on a $1 \mathrm{~km} \times 1 \mathrm{~km}$ field for 75 and 100 nodes. All the nodes are distributed uniformly within the above fields, respectively. The number of channels used here is three and communication range (CR) is $180 \mathrm{~m}$. The tree is constructed so that the path from any node to the coordinator is the shortest path based on the hop count. The physical interference model relationship is constructed through the definition of interfering parent as in [3], [4]. The simulation is conducted with 6 different topologies for each network size. The different topologies would lead to the different total number of timeslots required. In order to achieve consistent evaluation, we ensure that the total number of hops in any two topologies differs by less than 5 percent. 


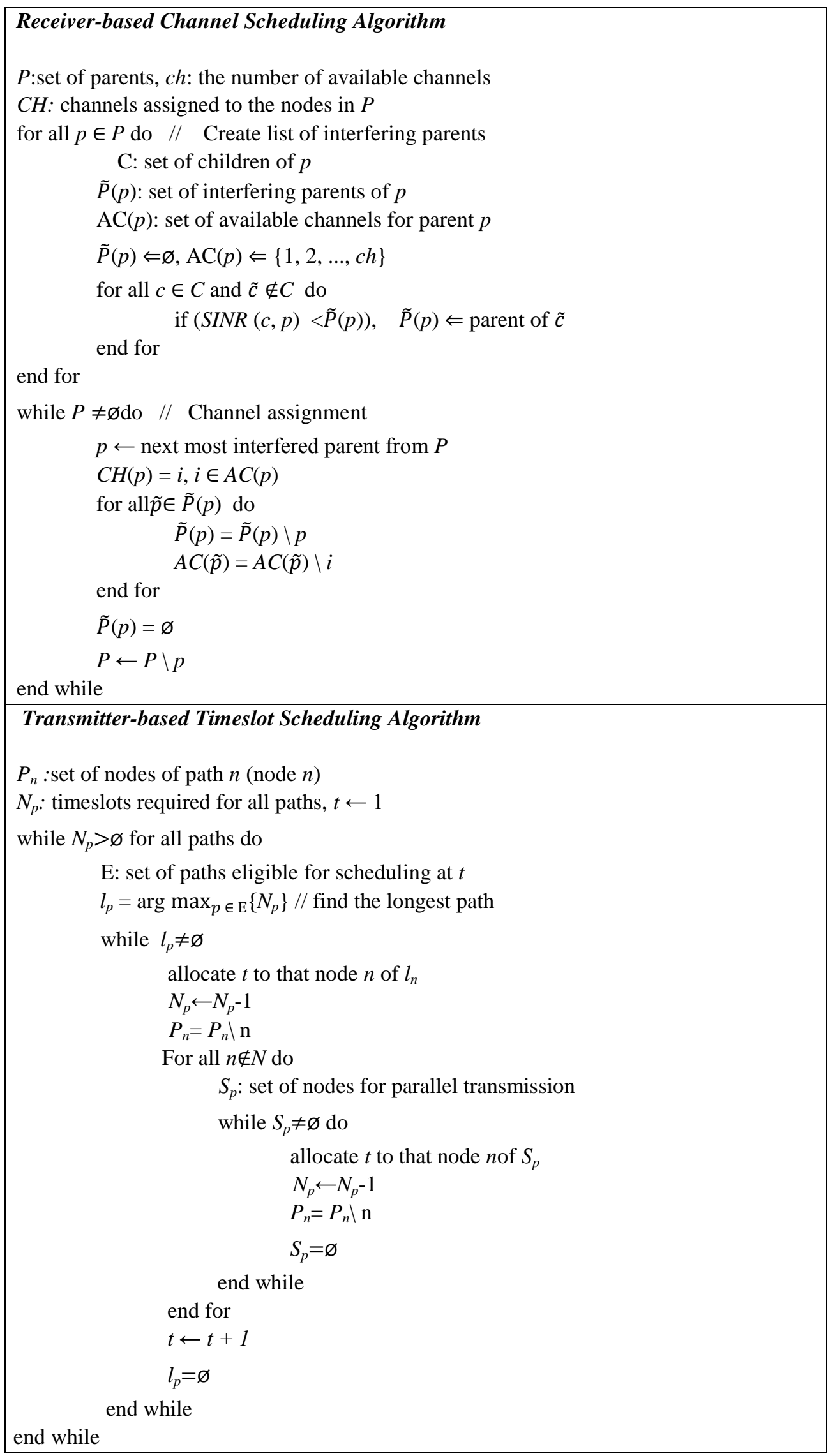

Figure 3. The Pseudo Codes of Scheduling Algorithms 
In Figures 4-5, we compare the e2e delay of our e2e delay-based algorithm to [3], which is non-e2e delay-based. We plotted the cumulative distribution function (CDF) with the maximum e2e delay for both the algorithms among the 6 different topologies. The CDF shows that our approach reduces the e2e delay in all network sizes. Although the reduction in the maximum e2e delay achieved by our approach is just 3 timeslots for 25-node network, the reduction in the e2e delay is more than 30 timeslots when the network size is increased to 100. Through simulation, we observe that although simultaneous transmissions reduce the number of timeslots by allowing other nodes to reuse the same timeslots, they also deteriorate the e2e delay because simultaneous transmissions prevent timeslots from being allocated in a staggered sequence.

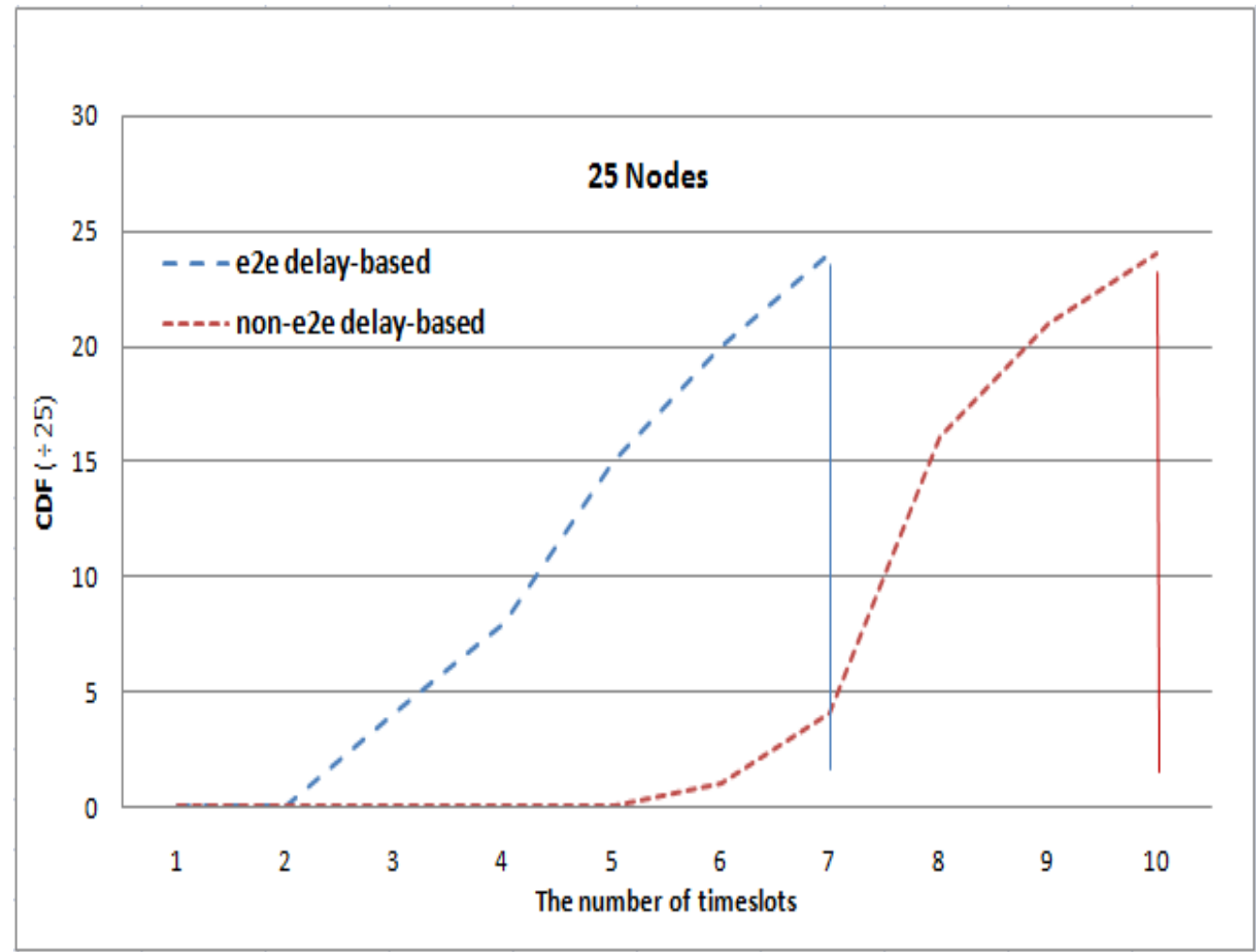

Figure 4. Cumulative Distributions of e2e Delay-based and Non-e2e Delay-based Algorithms for 25 Nodes 


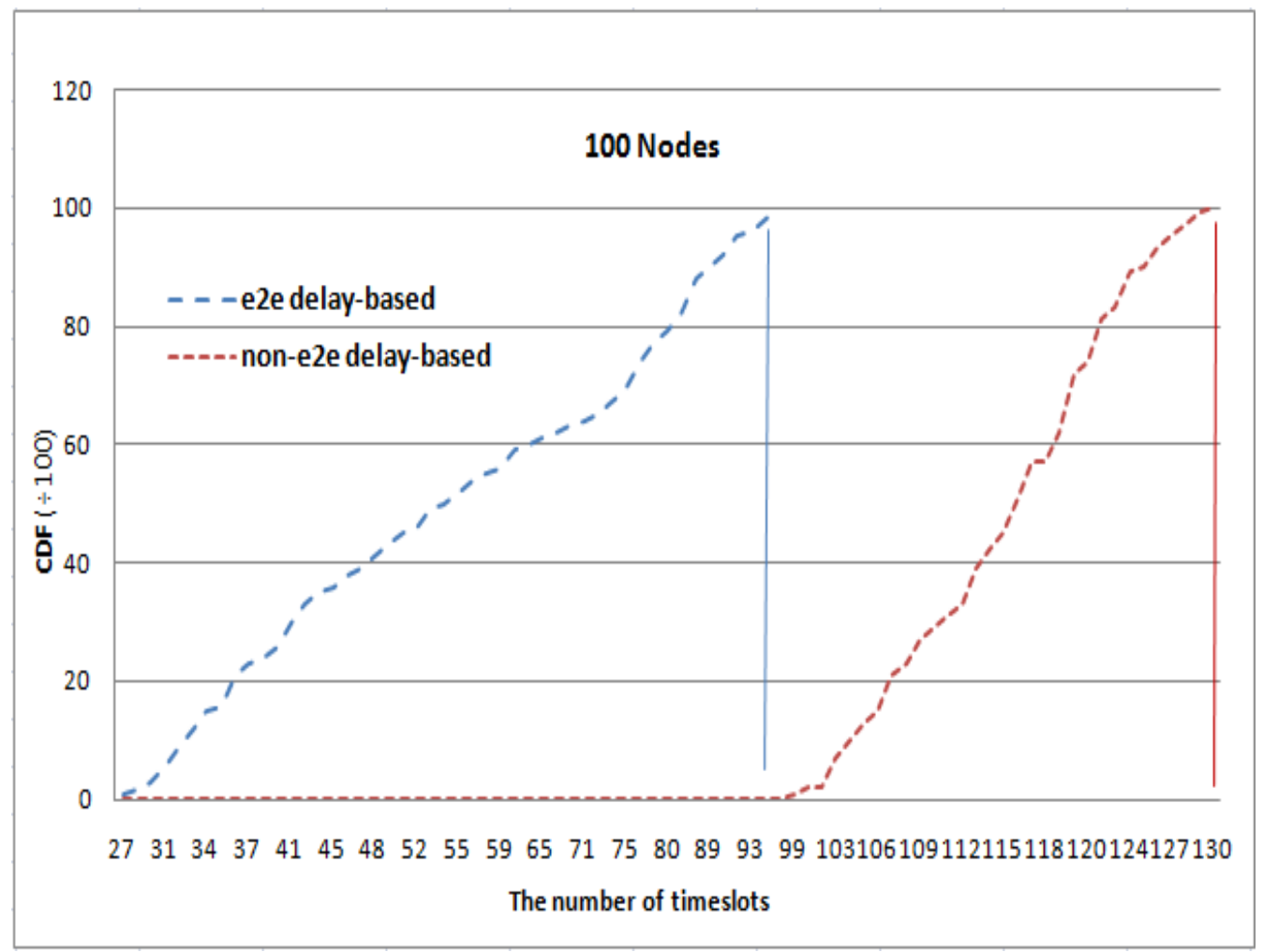

Figure 5. Cumulative Distributions of e2e Delay-based and Non-e2e Delay-based Algorithms for 100 Nodes

\section{Conclusion}

In this article, we propose a novel scheduling algorithm using multi-channel and multi-timeslot with the objective of reducing the end-to-end (e2e) delay in a treebased wireless sensor network. The algorithm exploits a staggered timeslot allocation in terms of e2e paths, rather than the one with individual branches. It also increases simultaneous data transmissions. Our proposal achieves substantial improvement on the e2e delay over current studies that focus on minimizing the number of timeslots, rather than e2e delay.

\section{References}

[1] Part 15.4: Low-Rate Wireless Personal Area Networks (LR-WPANs) Amendment 1: MAC sub-layer, IEEE Standard 802.15.4e, (2012).

[2] T. R. Park, Y. G. Kim, M. J. Lee and J. S. Chae. "Multi-hop extension for ieee 802.15.4e" IEEE 802.1515-08-0422-03-004e, (2008).

[3] O. D. Incel, A. Ghosh, B. Krishnamachari and K. Chintalapudi, "Multi-Channel Scheduling for Fast Convergecast in Wireless Sensor Networks", USC CENG Technical Report CENG, (2009).

[4] O. D. Incel, A. Ghosh, B. Krishnamachari and K. Chintalapudi, "Fast Data Collection in Tree-Based Wireless Sensor Networks", IEEE Transactions on Mobile Computing, vol. 11, no. 1, (2012), pp. 86-99.

[5] Y. G. Kim and M. J. Lee, "Scheduling Multi-channel and Multi-timeslot in Time Constrained Wireless Sensor Networks via Simulated Annealing and Particle Swarm Optimization," IEEE Communication Magazine, vol. 52, no. 1, (2014), pp. 122-129. 


\section{Authors}

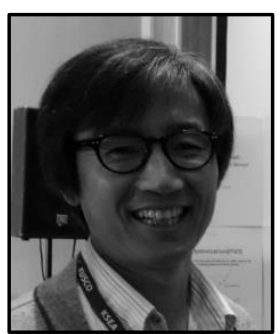

Yang G. Kim, he received a Ph.D. degree in Electrical Engineering from the CUNY Graduate Center. He is currently as an Adjunct Assistant Professor in the Department of Computer Engineering Technology at New York City College of Technology. His research interest includes MAC and routing in wireless networks and optimization for scheduling resources in wireless personal area networks and home networks.

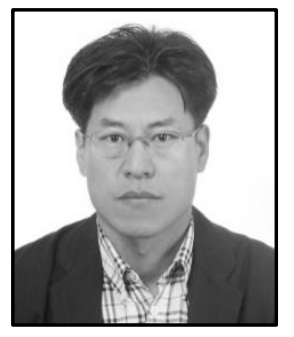

ByoungSeob Park, he received his Ph.D. degree in computer science and engineering from Sogang University, Seoul, Korea in 1997. He is currently a professor at the Department of Computer Systems \& Engineering, Inha Technical College, Incheon, Korea. His research interests include Wireless sensor network, IoT (Internet of Things), and Mobile healthcare services.

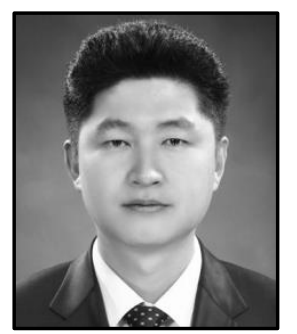

Hyo Hyun Choi, he received his Ph.D. degree in computer science and engineering from Sogang University, Seoul, Korea in 2005. He is currently an associate professor at the Department of Computer Science, Inha Technical College, Incheon, Korea. His research interests include wireless sensor network, unmanned aerial vehicle swarm control, internet of things and wireless mesh routing protocol. 
International Journal of Future Generation Communication and Networking Vol. 9, No.7, (2016) 